\title{
Buried AGNs and Starbursts in Luminous and Ultra-Luminous Infrared Galaxies
}

\author{
João Rodrigo S. Leão ${ }^{1}$, Patrícia Hepp ${ }^{1}$, and Claus Leitherer ${ }^{2}$ \\ ${ }^{1}$ Institute for Mathematics and Physics, Universidade Federal do Rio Grande - FURG \\ C.P. 476, Av. Itália, Rio Grande - RS, Brazil \\ Email: jrsleao@gmail.com \\ ${ }^{2}$ Space Telescope Science Institute, 3700 San Martin Drive, Baltimore, MD 21218, USA \\ Email: leitherer@stsci.edu
}

\begin{abstract}
We present the first results of a Spitzer Space Telescope survey of 28 LIRGs and ULIRGs. We used infrared emission lines to separeate AGN and starburst powered systems. We find strong evidence that the incidence of nuclear activity increases with infrared luminosity.
\end{abstract}

Keywords. galaxies: active, galaxies: starburst, infrared: galaxies

We present the first results of a Spitzer Space Telescope survey of 28 luminous $(11<$ $\log L_{\mathrm{IR}} / L_{\odot}<12$; LIRGs) and ultra-luminous infrared galaxies (with $\log L_{\mathrm{IR}} / L_{\odot}>12$; ULIRGs). Many of these galaxies are found in pairs and are powered by either nuclear activity or star formation (Sanders \& Mirabel 1996). The study of Sanders et al. (1988) finds evidence that the fraction of AGNs increases with the luminosity of the host galaxy.

The IRS spectrograph onboard the Spitzer Space Telescope was used in to cover the 4-35 $\mu \mathrm{m}$ wavelength range at low resolution. The goal is to use near-infrared emission lines to separate AGNs from starbursts.

The images were used to assign a marger stage to each galaxy. Precise galaxy separation measurements were performed. The IRS spectra show an enormous variety in shapes and detected emission and absorption lines. These lines were used to separate galaxies dominated by AGN activity, star formation, or both. Most AGNs are also advanced mergers. This is in agreement with the Sanders et al. (1988) scenario to explain LIRGs and ULIRGs.

We find that for LIRGs, starbursts dominate at $75 \%$ of the total (the remaining $25 \%$ are AGNs). For ULIRGs, AGNs and composite systems dominate, constituting $92 \%$ of the sample. Compared with optical studies, we thus find even stronger evidence for so-called luminosity dependence. Based on this small sample, we find no evidence that advanced mergers should be preferentially found in more luminous systems. In other words, we cannot claim that the infrared luminosity is a precise tracer of merger activity.

\section{References}

Sanders, D. B. \& Mirabel, I. F. 1996, ARAA, 34, 749

Sanders, D. B., Soifer, B. T., Elias, J. H., Neugebauer, G., \& Matthews, K. 1988, ApJ, 328, 35 